\title{
Neural Networks and Machine Learning for Pattern Recognition
}

\author{
Arafat A. Muharram \\ Associate Professor \\ Department of Computer \\ Science \\ Faculty of Computer Science \\ and Engineering \\ Hodeidah University, Hodeidah, \\ Y.R.
}

\author{
Khaled M. G. Noaman \\ Associate Professor \\ Department of Distance \\ Learning \\ Deanship of E-Learning and \\ Distance Learning, \\ Jazan University \\ Jazan, Kingdom of Saudi \\ Arabia, K.S.A
}

\author{
Ibrahim A.A. Alqubati \\ Professor \\ Department of Information \\ Systems \\ Community College Najran \\ University \\ Najran, Kingdom of Saudi \\ Arabia, K.S.A
}

\begin{abstract}
This paper represents an application study for using the Neural Networks and Machine Learning to recognize the English alphabet (A-Z) through the use of pattern recognition techniques in image processing and specifically to the application of Neural Networks and machine learning as a matrix two dimension.
\end{abstract}

We used two techniques ANN and ML to compare their efficiencies and accuracies. We got $86.92 \%$ for ANN and $91.2 \%$ for ML.

\section{General Terms}

Pattern Recognition, Image Processing.

\section{Keywords}

Neural Networks, Machine Learning, Image Processing, Pattern Recognition.

\section{INTRODUCTION}

Using the ANN to recognize images is of great importance to researchers in the ANN field. It is worth noting that the different shapes of images and their contents as well as dealing with the ANN to recognize images are so necessary for researchers that they have to process these images through the stages that these images have to pass through i.e. clearing the images (filtering), minimizing or maximizing the images (scaling), the movement of the image at a specific angle (rotation),segmenting the images (segmentation),etc, depending on the nature of the image [1].

Since the applications on one of the ANNs i.e. back propagation which is used in this paper require data representation, that is meant for training in a particular way that helps in the ANN learning .It is necessary of course after the processing of the image used for training the ANN to represent the pixels value by the bipolar form $(1,-1)$, which is one of the popular forms.

After testing the ANN, we get the text file that contains the letters of the English alphabet[13 ].

In fact the text file that we get reduces the store size of the said letters.

That is to say, each pixel consists of four bytes, whereas in the text file each letter consists of one byte only.
It is also important to mention here that the structure of the ANN was designed keeping in mind the number of neurons and layers as well as the weights etc. Furthermore the program was written in a way that matches the designed structure of the ANN.

The second technique we used was Machine Learning. The Machine Learning field evolved from the broad field of Artificial Intelligence, which aims to mimic intelligent abilities of humans by machines.

An important task in Machine Learning is classification. Machine Learning in this paper was used to recognize the shape of the character, which depend on the black pixels taken individually, especially those in the interior of the objects shown. They don't carry any information whether they are part of a representation of an object or another, it is their spatial interrelations which make us recognize the shape and to create variations such as fonts of different boldness of the object[2][7].

\section{2. RESEARCH METHODOLOGY}

\subsection{Image Processing}

The techniques used in the image processing are as follows:

\subsubsection{Segmentation}

This means dividing the image into small parts that are the constituents of the original images. Since this paper aims at the optical character recognition (OCR), if one image contains two characters (A, B), the segmentation of this image will result in two separated images (small parts) for (A), (B) individually.

\subsubsection{Filtering}

Filtering is of great importance as it vanishes the scattered spots as well as the noise found in the image. Thus we get a clean and pure image.

In the present paper, I have used "Median" method to clear the image. This requires taking specific areas of the image with square dimension (2D),

following up the values of all the pixels, arranging them, taking their median and replacing all the (pixels) by the median pixel [6] .

\subsubsection{Image Minimization}


The size of the original image gets minimized and is used for saving a big area either in the memory or the hard disk. There are many ways to do so. Used was the following method :

\subsubsection{The Average Method}

We take the pixels available in specific square areas ) $\mathrm{n} n * \mathrm{n}$ ) of the original image and get the average of each of them, so that we can get a smaller area containing these averages.

\section{ARTIFICIAL NOURAL NETWORKS \\ 3.1 Backpropagation Algorithm}

Backpropagation consider one of high ability algorithms which use in classification. We have select this algorithm because of the best results in classification using ANN[10], specially that the second comparison method is ML(Decision Tree Learning ) which usually give precise results in classification .

Designed network with Backpropagation Algorithm for one hidden layer[5] and its parameters with which BP was in stability stage:

Input neuron 25

Hidden neuron 8

Output neuron 5

Initial weight in the rang $[-0.1,0.1]$

Learning Rule : Standard Delta Rule

Learning Parameter 0.5

Transfer Function : Hyperbolic Tangent (TanH)

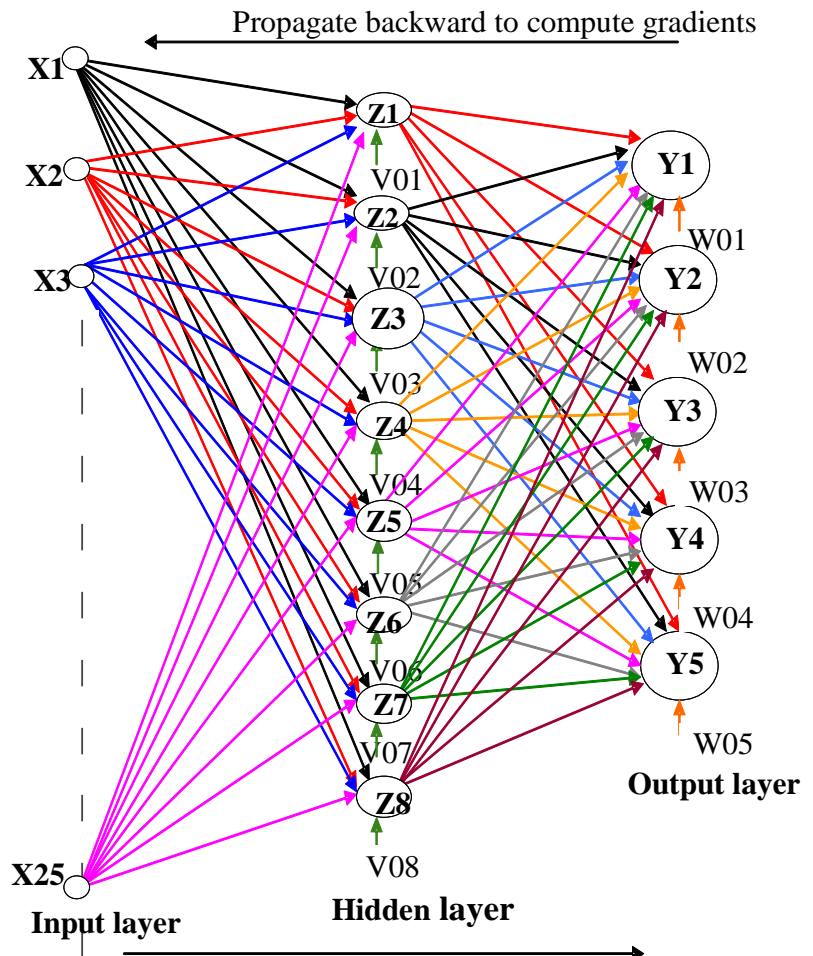

Propagate forward to compute outputs

Fig.1 Backpropagation Structure

\section{MACHINE LEARNING}

Machine Learning is inherently a multidisciplinary field. ML draws on results from probability, statistics, artificial intelligence, computational complexity theory ,control theory ,information theory ,philosophy and others fields.

Ml describe a variety of learning paradigms, algorithms, theoretical results and applications. Key idea are presented from various mentioned fields using a non specialist's vocabulary, with unfamiliar terms and concepts introduced as the need arises.

The learned function is represented by a decision tree. Learned trees can be represented as a sets of if-then rules to improve human readability. Each node in the tree specifies a test of some attribute of the instance [4][12].

We tried to classify English characters according to the features of it's shape and build a tree to separate the determinated alphabets group to two groups and continue using another features to reach the final nod which are the leaf of the tree contain the alphabet.

English text features are:

|- \(

| left ,middle, right

- up ,middle, down

। left, right, small, large

( left , right, up ,down

We tried by counting these features to know how to match the character by it's features regarding to it's shape.

The following example illustrates how we made ML technique to match character shape features

Example:

\section{E H B M A C O Z}

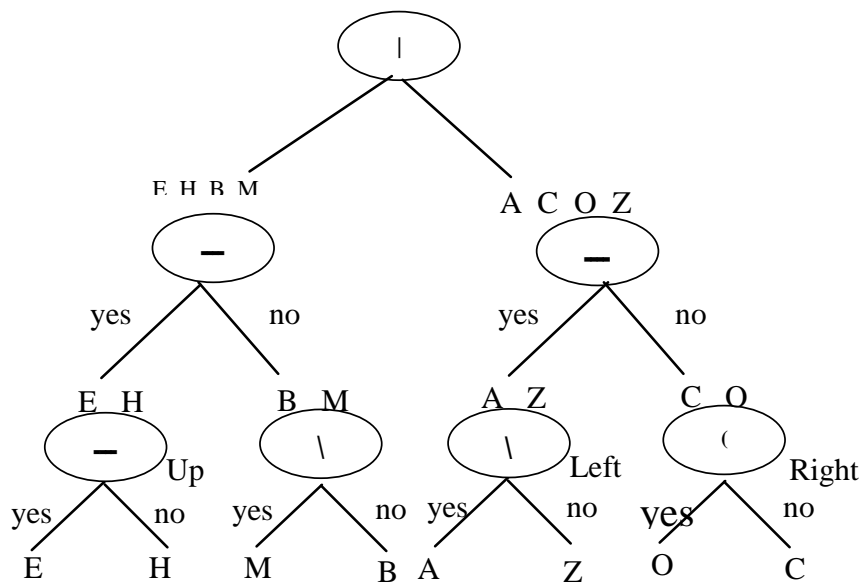

\subsection{Data Sets}

The data are images contain the 26 alphabets with 10 examples for every character with different shape.

Every character after image processing will be converted to array of pixels, we used array of 5 rows and 5 columns as standard for our work as follow: 


A
\begin{tabular}{|l|l|l|l|l|}
\hline-1 & -1 & 1 & -1 & -1 \\
\hline-1 & 1 & -1 & 1 & -1 \\
\hline 1 & -1 & -1 & -1 & 1 \\
\hline 1 & 1 & 1 & 1 & 1 \\
\hline 1 & -1 & -1 & -1 & 1 \\
\hline
\end{tabular}

\begin{tabular}{|l|l|l|l|l|}
\multicolumn{6}{c}{$\mathrm{A}$} \\
\hline-1 & -1 & 1 & -1 & -1 \\
\hline-1 & 1 & -1 & 1 & -1 \\
\hline 1 & -1 & -1 & -1 & 1 \\
\hline-1 & 1 & 1 & 1 & -1 \\
\hline 1 & -1 & -1 & -1 & 1 \\
\hline
\end{tabular}

\section{EXPERIMENTS DESIGN}

we have two experiments for different technique, one for Artificial Neural Networks and one for Machine Learning.

The Artificial Neural Network experiment and Machine Learning experiment, we applied in this paper for classification.

The input for the two experiments are the data sets mentioned above.

We have used Matlab 7.0 package for back-propagation method and self-made code for Decision Tree Learning method.

\section{RESULT AND DISCUSSION}

Through the use of image processing techniques, the letters of the English alphabet (A-Z) were changed into pixels depending on the number of which the ANN was designed, trained and tested.

It was clear that the number of pixels representing each letter depends on the letter, the kind and size of the font used. The ANN was capable of recognizing the letters through twenty five pixels for each letter with different fonts.

The Machine Learning ( Decision Tree Method ) which we used in this paper assigns a Boolean classification (yes or no) for the English alphabet (A-Z), beginning a disjunction of conjunctions of constraints on the attribute values of instance. Each path from the tree root to a leaf corresponds to a conjunction of attribute test, and the tree it self to a disjunction of these conjunctions

The table1 below shows the number of character recognize correctly by the techniques used.

Table 1. The Average Results for two Techniques ANN and ML

\begin{tabular}{|c|c|c|}
\hline Characters & ANN & ML \\
\hline A & 8 & 9 \\
\hline B & 9 & 9 \\
\hline C & 8 & 8 \\
\hline D & 8 & 10 \\
\hline E & 10 & 10 \\
\hline F & 9 & 10 \\
\hline G & 10 & 9 \\
\hline H & 9 & 10 \\
\hline I & 10 & 10 \\
\hline J & 8 & 9 \\
\hline K & 8 & 9 \\
\hline L & 9 & 10 \\
\hline M & 8 & 9 \\
\hline N & 8 & 9 \\
\hline O & 10 & 8 \\
\hline
\end{tabular}

\begin{tabular}{|c|c|c|}
\hline P & 9 & 8 \\
\hline Q & 8 & 8 \\
\hline R & 10 & 9 \\
\hline S & 8 & 8 \\
\hline T & 8 & 9 \\
\hline U & 10 & 8 \\
\hline W & 8 & 9 \\
\hline X & 8 & 10 \\
\hline Y & 8 & 10 \\
\hline Z & 8 & 9 \\
\hline Average & 86.92 & 9.12 \\
\hline
\end{tabular}

Table 2. Summary Testing Results of used methods

\begin{tabular}{|c|c|c|}
\hline \multirow{3}{*}{$\begin{array}{l}\text { Testing } \\
\text { Criterion }\end{array}$} & \multicolumn{2}{|c|}{ Method } \\
\hline & M L & NNs \\
\hline & $\begin{array}{l}\text { Correctly } \\
\text { classified } \\
\text { instances in }(\%)\end{array}$ & $\begin{array}{l}\text { Correctly } \\
\text { classified } \\
\text { instances in }(\%)\end{array}$ \\
\hline $\begin{array}{l}\text { Testing } \\
\text { Result }\end{array}$ & 86.92 & 91.2 \\
\hline
\end{tabular}

The correctly classified instances of the experiments are summarized in table 2,which shows that Machine Learning is able to increase the specificity from $80 \%$ by use of Neural Networks up to $91.2 \%$.

\section{CONCLUSION}

Most of the problems that get solved in the ANN ,Machine Learning, specifically, those in the form of images have to pass by many stages to be changed into other forms by using methods on scientific bias in image processing in accordance with which it becomes possible to design the hierarchy of the ANN, Machine Learning followed by the training and testing.

This shows that image processing is the basic entrance and starting point of some of the ANN and Machine Learning applications. This makes it necessary for researchers in these fields to take care of and have a thorough knowledge of the image processing techniques and translating them into application programs with the purpose of getting a new representation for images that matches the ANN and Machine Learning nature.

The results which we got in this paper show that the Machine Learning gives higher accuracy than ANN, maybe because of two reasons, first one is the nature of the characters features which are different from one character to another, the second one is the experiment of Artificial Neural Network, since Neural Network has two faces one for training and another for testing. For the both methods we applied two different input,but for Machine Learning we have just one face (classification) only.

\section{AUTHOR PROFILES}

Arafat A. Muharram received the $\mathrm{PhD}$ degree in Business Administration (Computer Networking management) Commerce Research Center from Pune university, India, in 2007. 
Currently, he is an associate professor at Hodeidah University, Hodeidah, Yemen R.

Khaled M. G. Noaman received the $\mathrm{PhD}$ degree in Artificial Intelligent (artificial neural networks) from Wroclaw University of Technology, Wroclaw, Poland, in 1999. In July 2006 he got promoted to associate professor at the Faculty of Computer Science and Information Technology, Sana'a University, Sana'a, Yemen. From 2012 till now he is working in the department of Distance Learning, Deanship of E-Learning and Distance Learning, Jazan University, Jazan, Kingdom of Saudi Arabia.

Ibrahim A. A. Alqubati received the Msc degree in computer science from Wroclaw University of Technology,Wroclaw,Poland, in 1990 and the $\mathrm{PhD}$ degree in Artificial Intelligent (artificial neural networks) from Wroclaw University of Technology, Wroclaw, Poland, in 1999. He is a Professor at the Faculty of Computer Science and Engineering, Hodeidah University, Hodeidah, Yemen, Currently he is a Professor at Community College of Najran University, Najran , Kingdom of Saudi Arabia.

\section{REFERENCES}

[1] Anil K. Jain, Fundamentals of Digital image Processing, Prentice Hall, USA, 1989

[2] Cosmin Grigorescu, Student Member, IEEE, and Nicolai Petkov: Distance Sets for Shape Filters and Shape Recognition, IEEE TRANSACTIONS ON IMAGE PROCESSING, VOL. 12, NO. 10, OCTOBER 2003

[3] Dorst, L. and A.W.M. Smeulders, Length estimators compared, in Pattern Recognition in Practice II, E.S. Gelsema and L.N. Kanal, Editors. 1986, Elsevier Science: Amsterdam. p. 73-80.
[4] Gunnar Ratsch, A Brief Introduction into Machine Learning, Friedrich Miescher Laboratory of the Max Planck Society , Germany, www.ccc.de/congress/2004/fahrplan/files

[5] Hirose, Y .,Back Propagation Algorithm which Variesthe Number of HiddenUnits, NeuralNetworks, Vol.4,pp.6166,1991

[6] Huang, T.S., G.J. Yang, and G.Y. Tang, A Fast TwoDimensional Median Filtering Algorithm. IEEE Transactions on Acoustics, Speech, and Signal Processing, 1979. ASSP-27: p. 13-18.

[7] JACKY HERZ and ROGER D. HERSCH: Analyzing character shapes by string matching techniques, Electronic Publishing, VOL. 6(3), 261-272 (SEPTEMBER 1993)

[8] Mickey Williams, David Bennett, et al., Visual C++6 Copyright 2000 by sams publishing, USA

[9] .Pavis Chapman, teach yourself Visual $\mathrm{C}++6$ in 21 days.2000 ,New Delhi

[10] R.Schalkoff ,Artificial Neural Networks, McGrawHill,1999.

[11] Russ, J.C., The Image Processing Handbook. Second ed. 1995, Boca Raton, Florida: CRC

[12] TOM M. MITCHELL, Machine Learning ,McGraw-Hill, USA, 1997

[13] WhiteH,ArtificialNeuralNetworks:ApproximationandLea rningTheory,Blackwell,1992. 